\title{
The Application of Up-conversion Nanoparticles Materials in Cancer Diagnosis and Therapy
}

\author{
Ya-juan XU1 ${ }^{1}$, Tao LUAN² ${ }^{2}$, Bai-tao ZENG ${ }^{2}$ and Wen-shu LIU ${ }^{3^{*}}$ \\ ${ }^{1}$ Oral and maxillofacial surgery, Jilin provincial tumor hospital, Changchun, China \\ ${ }^{2}$ College of life science and technology, Changchun University of Science and \\ Technology, Changchun, China \\ ${ }^{3}$ Oral cavity hospital, the first clinical hospital of jilin university, Changchun, China \\ * Corresponding author: 1252042806@qq.com
}

Keywords: Up-conversion nanoparticles, Tumor detection, Oncotherapy, Photo-d ynamic therapy.

\begin{abstract}
According to estimates by oncologists, if early tumor diagnosis and treatment in a timely manner can be achieved in the millimeter level or below, the cure rate of cancer will be able to increase to $80 \%-90 \%$. With the rapid development and maturity of nano technology and scientific theory, Up-conversion nanoparticles (UCNPs) have become a tumor nanoplatform which can be used for early diagnosis and treatment.The review summarizes the progress of Up-conversion nanoparticles in detection and treatment of tumor in some research.
\end{abstract}

\section{Introduction}

Nano medical oncology, is not only a new field of nano medicine, but also a current cutting-edge technology in the central areas which countries give an priority to the develop. A research of the National Cancer Institute (NCI) indicated that the development of nano in biomedical security can provide a new idea to explore technological nano probe, that is specific, efficient and intelligent method which can use technological nano probe to contribute tremendously to cancer prevention, imaging, diagnosis and treatment [1,2].Fluorescent probe with nano material as carrier has been widely used in the field of biochemistry, especially in the diagnosis and treatment of tumors. The size of the nanoparticles may be smaller than the cancer cells about 100 times, even 1000 times, so they can easily get through the barrier of cancer cell. What's more, they will preferentially accumulate around the active tumor tissue in the capillaries and unsound system of lymphatic drainage causing enhanced permeability and retention effect (EPR)[3].

\section{Tumor Diagnosis}

\section{Imaging of Tumor}

Molecular imaging of targeted tumor utilized the expression differences between the normal cells and tumor cells, it is a tumor detection technology and used as target because of the features of specific recognition by molecular probe, it plays a very important role in the diagnosis and prognosis of tumor.

The unique optical properties of Upconversion nanoparticles (UCNPs)with tombarthite has been extensively used in the active imaging of tumors. In 2009, Li Fuyou's team from the Fudan University used the RGD sequence combine with UCNPs, firstly tumor targeted imaging was realized based on UNCPs.RGD peptides 
can combine with av|33 integrin receptors that highly expressed in the surface of tumor angiogenesis or malignant cells, combination's activity is about 170 times more than the same kind of linear polypeptide. The nude mices with Hela tumor cells were injected intravenously with FA-UCNPs for one day, the obvious upconversion fluorescence signal(UCL) was detected at the tumor site, but in the control group was not detected signal. The experimental results show that the RGD peptides is successfully connected to the UCNPs surface, and UCNPs are able to efficiently target the glioma tumor [4]. Later, they will be a wide variety of tumor cells with a high affinity neurotoxin connected to UCNP surface, also realized the targeted UCL imaging. This work shows the UCNP probe in the diagnosis of tumor cells can be used as a molecular target [5-7].The Gu group by a new kind of polymer molecules FASOC photosensitizer molecule zinc phthalocyanine ( $\mathrm{ZnPc}$ ) loaded into UCNP surface, and marked with folic acid molecular target tumor, building a set of diagnosis and treatment of nanometer platform integration[8].

\section{Multimode Imaging of UCNPs}

At present, it is reported that three ways to use UCNP for biological imaging including Magnetic Resonance Imaging (MRI), X-ray Computed Tomography (CT), Positron Emission Tomography(PET), Single Photon Emission CT(SPECT) and Ultrasound(UCL). With a high sensitivity, optical imaging is limited because the depth of penetration is not enough in the biological tissue. On the contrary, MRI has the deeper depth of penetration and the higher spatial resolution, but its imaging sensitivity is poor.The high resolution 3D structure can be reconstructed by Computed Tomography (CT) Imaging, its sensitivity of imaging is low in soft tissue . The majority of UCNP used in biological imaging is to integrate several kinds of imaging technology through the design of the core shell structure, which can be used for a deeper and more comprehensive understanding of the tumor.

Recently, the construction of UCNPs based PET/MRI/UCL or CT/MRI/UCL three mode imaging probes has become the trend of development. Li subject group using a cationic auxiliary ligands self-assembly method, using folic acid as a boot to the unit, ucnps as UCL imaging unit construct UCL/PET/MRI tri modal target to imaging probes, and successfully applied to target tumor cells to UCL/PET/MRI tri modal imaging. [10].In 2014, Li Fuyou's group synthesized UINCP(NaLuF $\left.{ }_{4}: \mathrm{Yb}, \mathrm{Tm}\right)$, then coated the NaGdF4 on its surface and doped 153SM which has 46.3 hours' half-life, CT/SPECT UCL/MRI/ four mode imaging is realized.[11].

\section{Tumor Treatment}

\section{Photodynamic Therapy of UCNPs}

Photodynamic therapy(PDT) is the alternative treatment technology which has developed rapidly in the recent years. Photodynamic therapy (PDT) requires three elements: a specific wavelength of light, the photosensitizer and oxygen molecules in cells. The principle of photodynamic therapy (PDT) is that under the light, the photosensitizer starts up photochemical reaction to generate singlet oxygen or reactive oxygen free radicals via absorption of specific wavelengths of light, then oxidatively damage to the surrounding tumor cells. Compared to the general method of tumor therapy, photodynamic therapy has a better selectivity, which can control treatment area and degree of exposure by controlling the position and energy of light source, without harming the surrounding normal tissues; no drug accumulation toxicity, other therapies can be used simultaneously and treatment can be 
repeated[12].In 2011, Liu Zhuang's group transferred UNCP to the aqueous phase via amphiphilic copolymer C18PMH-PEG, and then loaded photosensitizer Ce6. At the same time, using fluorescence of up-conversion $550 \mathrm{~nm}$ and $660 \mathrm{~nm}$ to label the tumor site of rat, constructed UNCP with dual function of treatment and imaging. And they made 4T1 breast tumor Balb/c rat animal model. UCNP- Ce6 was administered to tumor by intratumoral injection method, then via near-infrared laser irradiation at $980 \mathrm{~nm}$, for the first time realized photodynamic therapy based on UCNP in vivo.Recently, the research group first designed and constructed PDT system with $\mathrm{pH}$ responsiveness. They loaded CE6 to $\mathrm{pH}$ sensitive UCNPs. Under acidic condition of the tumor microenvironment stimulation, prepared Ce6-1JCNPs system surface charge changes from negative to positive, thus promotes accumulation of Ce6-UCNPs in the tumor tissue. Dual modality imaging navigation of tumor oriented photodynamic therapy was realized[13].In 2012, Kong Xianggui research group from Changchun Institute of Optics, Fine Mechanics and Physics first detected the complexes of up-conversion nanoparticles and photosensitizer producing 1270 $\mathrm{nm}$ fluorescence emission of singlet oxygen. They conducted UCNP with the small hydrophilic molecule AEP by ligand exchange, and loaded photosensitizer (Rose Bengal) to the surface of the UCNP by covalent coupling for the first time. Fluorescence resonance energy transfer at $540 \mathrm{~nm}$ fluorescence of UCNP $\left(\mathrm{NaYF}_{4}\right.$ : $\mathrm{Yb}^{3}+, \mathrm{E}^{\mathrm{r} 3+)}$ and photosensitizer can produce singlet oxygen to kill tumor cells. Loading photosensitizer can shorten direct distance of the donor and acceptor effectively by covalent coupling, and improve the efficiency of FRET and increase the yield of singlet oxygen [14].

\section{Heat Treatment of UCNPs}

Photothermal treatment is the treatment of using light absorption materials produce heat to "burn" tumor cells directly under the light. Using nano probe to generate heat for the treatment of the tumors, is one of the research hotspots in the nanometer medicine. In 2003,J.Halas professor group from Rice University conducted coated $\mathrm{SiO}_{2}$ nanometer spherical shell material with human breast cancer cells in vitro culture, under the irradiation of near-infrared laser, gold nanometer spherical shell material converted luminous energy into thermal energy, making tumor cells occurs irreversible thermal damage[15].In the experiment of Wang Chao from Suzhou University, they use layer by layer assembly to synthesized multifunctional up-conversion nanophase materials (MFNP), the enrichment of MFNP in the tumor was increased under magnetic field, and dual modal imaging was performed via up-conversion fluorescence and T2 magnetic resonance imaging. The photothermal treatment for tumor was realized by using gold layer of MFNP under the guidance of imaging[16].PhD Chen Chunying and Wu Xiaochun group reported $\mathrm{Au} @ \mathrm{SiO}^{2}$ used as drug carrier for the first time. They package it on the gold nanorods mesoporous silica and solve the difficult problem that gold nanorods is not easy to carrying drugs, $\mathrm{Au} @ \mathrm{SiO}_{2}$ can located conveniently in cells by two photon imaging technology. After loading the anticancer drugs doxorubicin, under different laser, $\mathrm{Au} @ \mathrm{SiO}_{2}$ has realized the two kinds of tumor treatment mode: one is that low power laser induced cancer drugs doxorubicin releasing to form a pattern of chemotherapy, another is that high power laser light energy is converted into heat energy and the pattern of heat treatment and double direct implementation [17]. 


\section{Drug Transport and Gene Therapy}

UCNP not only can be phagocytosised nonspecifically by endocytosis, but it can modify its surface and corresponding target cell surface receptors, which makes up-conversion nanoparticles link certain cells specifically,therefore, UNCP can be a carrier of antitumor drugs according to its own fluorescence labeling target molecular. The superparamagnetic ferroferric oxide magnetic nano surface with covalently anchored photosensitizer and absorbed by cancer cells, apoptosis can be induced by light.In 2011, research group of Liu Zhuang used UNCP as a vector, segmented copolymer coating nanoparticles, loaded antineoplastic Doxorubicin in hydrophobic layer using hydrophobic interaction, controlled the release of drug by adjusting the $\mathrm{PH}$ value. Research group of Lin anti platinum precursor drug loading to UNCP surface and intravenous injection in vivo, UNCP emission of ultraviolet light in 980 $\mathrm{nm}$ near-infrared excitation will induce the platinum (IV) the divalent platinum, which inhibits tumor growth.Similar with package drugs, it's also available to load genes on the surface of magnetic nanoparticles on gene therapy.Professor Cui Daxiang group modified tree molecules on magnetic nanoparticles and loaded Survivin antisense oligonucleotide, successfully achieved the gene therapy.

\section{Summary and Forecasting}

UNCP has great potential in tumor detection and treatment, and has been paid more and more attention and research. Although UNCP has made great progress, but there are still many problems need to be solved, it restricts its universal application.

In photodynamic therapy process, we think that it is very low from nanoparticles to molecular photosensitizers fluorescence resonance energy transfer efficiency and has not been very clear in the mechanism, the low luminous efficiency of UNCP in vivo is still a bottleneck restricting its development and application. Although it can improve the luminescence efficiency by using the method of the doping of organic ground and the core shell coating for sensitization of ions and the luminescent ion, to improve the conversion efficiency of luminescence such as IR806.But how to enhance the absorption ability of the near infrared light, how to reduce the toxicity of the dye, it is still need to be further studied. Now based on huge investments, research results and a large number of governments in the research of nano - tumor medicine, it can will be predicted, Up conversion Luminescence probe will also provide a powerful tool for the diagnosis and treatment of tumors at various stages, and fundamentally change the status of the tumor diagnosis and treatment.

\section{References}

[1] Park K, Lee S, Kang E, Kim K, Choi K, Kwon IC. New generation of multifunctional nanoparticles for cancer imaging and therapy. Adv.Funct. Mater.19(2009)1553-1566.

[2] Song H, He R, Wang K, Ruan J, Bao CC, Li N, Ji JJ, Cui DX. Anti-HIF-1 [alpha] antibody-conjugated pluronic triblock copolymers encapsulated with Paclitaxel for tumor targeting therapy. Biomaterials. 31 (2010) 2302-2312.

[3] Liqin Xiong, Zhigang Chen, Qiwei Tian, Tianye Cao, Congjian Xu, Fuyou Li.High contrast upconversion luminescence targeted imaging in vivo using peptide-labeled nanophosphors. Anal Chem. vol. 81(2009) 8687-8694. 
[4] Xiong L. Q., Chen Z. G., Yu M. X., Li F. Y., Liu C., Huang C. H. Synthesis, characterization, and in vivo targeted imaging of amine-functionalized rare-earth up-converting nanophosphors. Biomaterials.30(2009) 5592-5600.

[5] Yu XF, Sun Z, Li M, Xiang Y, Wang QQ, Tang F et al.Neurotoxin-conjugated upconversion nanoprobes for direct visualization of tumors under near-infrared irradiation. Biomaterials.31(2010)8724-8731.

[6] Auzel F. Upconversion and anti-stokes processes with $\mathrm{f}$ and $\mathrm{d}$ ions in solids. Chemic Reviews. 104(2004)139-174.

[7] Liu C., Gao Z., Zeng J., Hou Y., Fang F., Li Y. et al. Magnetic/Upconversion Fluorescent NaGdF4:Yb,Er Nanoparticle-BasedDual-Modal Molecular Probes for Imaging Tiny Tumors in Vivo. Acs Nano. 7(2013) 7227-7240.

[8] S. Cui, D. Yin, Y. Chen, Y. Di, H. Chen, Y. Ma et al. In Vivo Targeted Deep-Tissue Photodynamic Therapy Based on Near-Infrared Light Triggered Upconversion Nanoconstruct. ACS Nano.7(2013)676-688.

[9] Wu, Y., Sun, Y., Zhu, X., Liu, Q., Cao, T., Peng,J. et al. Lanthanide-based nanocrystals as dual-modal probes for SPECT and X-ray CT imaging. Biomaterials. 35(2014)4699-4705.

[10] Sun, Y., Zhu, X., Peng, J.,Li, F. Core-Shell Lanthanide Upconversion Nanophosphors as Four-Modal Probes for Tumor Angiogenesis Imaging. ACS nano, 7(2014)11290-11300.

[11] Y. I. Park, J. H. Kim, K. T. Lee, K. S. Jeon, H. B. Na, J. H. Yu et al. Nonblinkingand Nonbleaching Upconverting Nanoparticles as an Optical Imaging Nanoprobeand T1 Magnetic Resonance Imaging Contrast Agent.Adv. Mater. 21(2009)4467-4471.

[12]Zhang P., Steelant W., Kumar M., Scholfield M.Versatile photosensitizers for photodynamic therapy at infrared excitation. Journal of the American Chemical Society. 129(2007) 4526-4527.

[13] Wang C, Tao H, Cheng L, Liu Z.Near-infrared light induced in vivo photodynamic therapy of cancer based on upconversion nanoparticles. Biomaterials, vol. 32 (2011) 6145-6154.

[14]Kai Liu, Xiaomin Liu, Qinghui Zeng, Youlin Zhang, Langping Tu, Tao Liu, et al. Covalently assembled NIR nanoplatform for simultaneous fluorescence imaging and photodynamic therapy of cancer cells. ACS nano. 6(2012)4054-4062.

[15] Gobin A M, Lee M H, Halas N J, James W D, Drezek R A and West J L. Near-infrared resonant nanoshells for combined optical imaging and photothermal cancer therapy. Nano Lett. 7(2007)1929-1934.

[16]Cheng L., Yang K., Li Y., Zen X., Shao M., Lee S.T., Liu Z. Multifunctional nanoparticles for upconversion luminescence/MR multimodal imaging and magnetically targeted photothermal therapy. Biomaterials. 33(2012)2215-2222.

[17]Zhang Z, Wang L, Wang J, Jiang X, Li X, Hu Z. et al.Mesoporous silica-coated gold nanorods as a light-mediated multifunctional theranostic platform for cancer treatment. Adv. Mater. 24(2012)1418-1423. 